\title{
Confusing elements in a COVID-19 patient
}

\author{
Vassili Panagides ${ }^{1}$, Eloi Prudhomme ${ }^{1}$, Claire Stein ${ }^{1}$, and Jennifer Cautela ${ }^{1}$ \\ ${ }^{1} \mathrm{APHM}$
}

June 22, 2020

\begin{abstract}
We herein present illustrative clinical images of a large intra cardiac thrombus of a 72 year old man presenting with a SARSCoV-2 viral infection.
\end{abstract}

\section{Confusing elements in a COVID-19 patient}

Vassili Panagides $^{1}, M D$; Eloi Prudhomme ${ }^{2}, M D$; Claire Stein ${ }^{2}, M D$, Jennifer Cautela ${ }^{3}, M D$

1 : Aix Marseille University, intensive care unit, Department of Cardiology, Hopital Nord, Marseille, France

2 : Assistance Publique - Hôpitaux de Marseille, Hôpital Nord, Médecine Intensive Réanimation 13015 Marseille, France

3 : Heart Failure and Valvular Heart Diseases Unit, Department of Cardiology, Mediterranean University Cardio-Oncology Center (MEDI-CO Center), Hôpital Nord, Aix-MarseilleI University, Marseille, France

Disclosure : None of the authors have any conflicts of interest regarding the submitted material

Short abstract: We herein present illustrative clinical images of a large intra cardiac thrombus of a 72 year old man presenting with a SARS-CoV-2 viral infection.

Image:

A 72-year-old man was referred for acute respiratory failure associated with cough and fever. He had a past medical history of chronic obstructive pulmonary disease and ischemic cardiopathy. His baseline medication included a dual anti platelet therapy. Few hours after his admission we noticed a rapid degradation of his lung function requiring oro-tracheal intubation and he was transferred to the intensive care unit. We performed a real-time reverse transcriptase-polymerase chain reaction of lower respiratory tract aspirate that was positive for SARS CoV-2. His chest computed tomography (CT) had typical manifestations of the disease with consolidation and reticular pattern, without visible pulmonary embolism. High fever motivate the realization of blood culture that showed a bacteremia of Staphylococcus lugdunensis. Thus, we performed a trans-esophageal echocardiography that showed two surprising elements. First, we discovered a thin structure in the anterior aortic sinus that could have been mistaken with a vegetation. It appears that it was the stent struts of the right ostial coronary artery (Panel $A$ and $B$ ) confirmed by CT. We also discovered a lobulated fixed mass which was related to the atrial septum (Panel $C$ and $D$ ). In the clinical context of SARS-CoV-2 infection, the main diagnosis suspected was a large thrombus. The severity of the patient did not allowed us to perform other exams to rule out any differential diagnosis. This interesting case highlights the serious pro-coagulable state in this devastating disease and the interest of repeating cardiac imaging even in the absence of pulmonary embolism $(1,2)$.

Legend:

Panel $A$ : Short axis view of the aortic valve 
Panel $B$ : Longitudinal view of the aorta

Panel $C$ : Pulmonary injected acquisition of the chest computed tomography

Panel $D$ : View of the interatrial septum

Ao : aorta; $L A$ : left atrium; $L V$ left ventricle; $R A$ : right atrium; $R V$ : right ventricle

References:

1. Connors JM, Levy JH. COVID-19 and its implications for thrombosis and anticoagulation. Blood. 2020 04;135(23):2033-40.

2. Bikdeli B, Madhavan MV, Jimenez D, Chuich T, Dreyfus I, Driggin E, et al. COVID-19 and Thrombotic or Thromboembolic Disease: Implications for Prevention, Antithrombotic Therapy, and Follow-up. J Am Coll Cardiol. 2020 Apr 15;

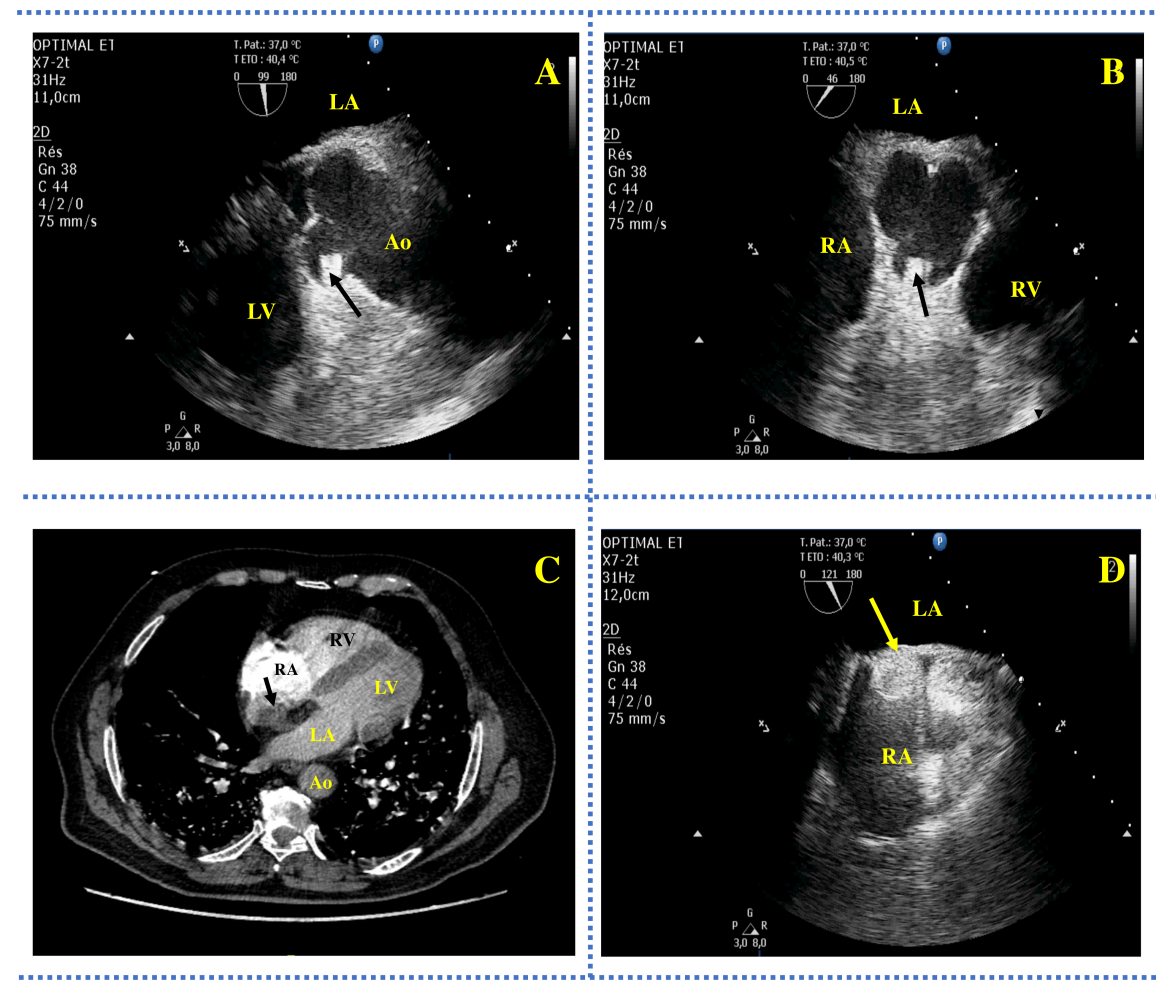

